\title{
BLIND RECEIVER FOR AMPLIFY-AND-FORWARD COOPERATIVE DIVERSITY SCHEME
}

\author{
Alexandre R. Fernandes ${ }^{(1)}$, André L. F. de Almeida ${ }^{(2)}$, Daniel B. da Costa ${ }^{(1)}$ \\ ${ }^{(1)}$ Department of Computer Engineering, Federal University of Ceará, Sobral, Brazil. \\ ${ }^{(2)}$ Department of Teleinformatics Engineering, Federal University of Ceará, Fortaleza, Brazil. \\ E-mails: alexandrefernandes@ufc.br, andre@gtel.ufc.br, danielbcosta@ieee.org
}

\begin{abstract}
In this paper, a new receiver that jointly and blindly estimates the channel parameters and the transmitted symbols in a multiuser cooperative diversity scheme is proposed. The receiver is based on the canonical polyadic (CP) decomposition of a tensor composed of received signals with dimensions associated with space (receive antennas), slot (cooperative channel) and time (symbol period). The receiver fits the tensor model using the Levenberg-Marquardt (LM) algorithm, assuming that channel state information (CSI) is not available at the relays neither at the base station. The performance of the proposed receiver is evaluated by means of computer simulations.
\end{abstract}

\section{INTRODUCTION}

Owing to the continuous necessity for higher throughput and increased data rates in wireless communication systems, in recent years there has been an upsurge of interest for cooperative diversity [1-5]. This technique has emerged as a promising wireless access solution that draws from the ideas of using the broadcast nature of the wireless channel to make communicating nodes help each other, of implementing the communication process in a distributed fashion and of gaining the same advantages as those found in multiple-input multiple-output (MIMO) systems. The basic idea is that mobile users relay signals for each other to emulate an antenna array and exploit the benefits of spatial diversity. The ultimate goal is to extend coverage without using high power levels at the transmitter, hence increasing connectivity and capacity. Several cooperative strategies have been proposed in the literature, such as the amplify-and-forward (AF), decode-and-forward (DF), and coded cooperation protocols. Among these, $\mathrm{AF}$ is one attractive cooperative protocol where the relay simply amplifies the signal received from the source and transmits the amplified signal to the destination, which has very low complexity and requires no decoding at relay nodes. Hence, AF relays are often preferable when complexity and/or latency issues are of importance. This paper primarily focuses on AF relaying systems.

On the other hand, the use of tensor decompositions has gained increased attention in several signal processing applications for wireless communication systems, specifically in situations where the received signals can be viewed as multidimensional variables [6-8]. The practical motivation for a tensor modeling

This work was partially supported by Pronex/ Funcap (Proc. 21.01.00/08). C. Alexandre. R. Fernandes is partially supported by FUNCAP/Brazil (Proc. BP1-0031-00106.01.00/10). André L. F. de Almeida is partially supported by $\mathrm{CNPq} /$ Brazil (Proc. 303238/2010-0). Daniel B. da Costa is partially supported by FUNCAP/Brazil (Proc. BP1-0031-00090.01.00/10). comes from the fact that one can simultaneously benefit from multiple (more than two) forms of diversity to perform multiuser signal separation/equalization and channel estimation under model uniqueness conditions more relaxed than with conventional matrix-based approaches.

In this paper, we propose a receiver that jointly and blindly estimates the channel gains, the antenna array responses and the transmitted symbols in a uplink multiuser cooperative communication system employing an antenna array at the destination (base station). We show that the received signals can be expressed as a tensor model called canonical polyadic $(\mathrm{CP})$ decomposition $[9,10]$, also known by parallel factor (or canonical) decomposition. The underlying tensor model has a powerful uniqueness property that allows the development of a blind receiver exploiting both spatial and cooperative diversities, with a great flexibility on the choice of some system parameters.

As far as the authors are aware, up to now there are only two works in the literature dealing with cooperative communications using a tensor-based approach $[11,12]$. However, in these works, there are no cooperative replicas of the transmitted signals that are combined at the receiver. In contrast, our work explicitly exploits cooperative diversity by incorporating the cooperative channels as an additional diversity dimension of the received data tensor.

The remainder of this paper is organized as follows. In Section 2, the system model is presented. Section 3 formulates the tensor modeling of the received signals and Section 4 presents the receiver algorithm. The performance of the proposed technique is evaluated by means of computer simulations in Section 5 , and Section 6 draws some conclusions.

\section{SYSTEM MODEL}

Let us consider that $M$ co-channel users transmit towards a base station employing an uniform linear array of $K$ half-wavelength spaced omnidirectional antennas. We consider a cooperative scenario where each user communicates with the base station through a direct link and with the help of $R$ relays. Each user and relay are equipped with a single antenna. We assume that fading is frequency-flat and that users are synchronized at the symbol level. The discrete-time baseband signal $x_{k, n}^{(S D)}$ received through the direct link (source-destination) at the $k$ th base station antenna and $n$th symbol period can be written as:

$$
x_{k, n}^{(S D)}=\sum_{m=1}^{M} h_{k, m}^{(S D)} s_{n, m}+v_{k, n}^{(S D)},
$$

where $h_{k, m}^{(S D)}$ is the channel linking the $m$ th user and $k$ th receive antenna, $s_{n, m}$ is the $n$th data symbol of the $m$ th user and $v_{k, n}^{(S D)}$ is the 
additive white Gaussian noise (AWGN) component at $k$ th antenna and $n$th symbol period. The data symbols $s_{n, m}, 1 \leq m \leq M$, are assumed to be independent and identically distributed (i.i.d.), with a uniform distribution over a quadrature amplitude modulation (QAM) or phase-shift keying (PSK) alphabet.

We consider that each user communicates with $R$ other users that work as amplify-and-forward relays multiplexed in time. This means that each relay amplifies the signal received from a user and forwards it to the base station (two-hop relaying) using a different time-slot. Moreover, it is assumed that a user and its $R$ relays are located in a cluster. This assumption implies that the signal received at a relay located within the cluster of the $m$ th user contains no significant contribution from the other users. Under these assumptions, the received signal $u_{r, m, n}$ at the $r$ th relay of the $m$ th user is then given by:

$$
u_{r, m, n}=h_{r, m}^{(S R)} s_{n, m}+v_{r, m, n}^{(S R)},
$$

where $h_{r, m}^{(S R)}$ is the channel gain between the $m$ th user and its associated relay (source-relay link) and $v_{r, m, n}^{(S R)}$ is the noise component.

Denoting $g_{r, m}$ as the amplification factor of the $r$ th relay of the $m$ th user, the signal $x_{k, r, n}^{(R D)}$ received at the $k$ th base station antenna during the $r$ th time-slot (relay-destination link) can be expressed as:

$$
x_{k, r, n}^{(R D)}=\sum_{m=1}^{M} h_{k, r, m}^{(R D)} g_{r, m} u_{r, m, n}+v_{k, r, n}^{(R D)},
$$

where $h_{k, r, m}^{(R D)}$ is the channel gain between $k$ th receive antenna and the $r$ th relay associated with the $m$ th user, and $v_{k, r, n}^{(R D)}$ is the corresponding noise component. Substituting (2) into (3), we get:

$$
x_{k, r, n}^{(R D)}=\sum_{m=1}^{M} h_{k, r, m}^{(R D)} h_{r, m}^{(S R)} g_{r, m} s_{n, m}+v_{k, r, n}^{(S D)}
$$

where

$$
v_{k, r, n}^{(S D)}=\sum_{m=1}^{M} h_{k, r, m}^{(R D)} g_{r, m} v_{r, m, n}^{(S R)}+v_{k, r, n}^{(R D)},
$$

denotes the overall additive noise contribution at the receiver, including the amplified noise at the relay.

In the following, we further work on the expressions (1) and (4) of the received signals $x_{k, n}^{(S D)}$ and $x_{k, r, n}^{(R D)}$, respectively, by including into the signal model some specifics of the propagation scenario. Let us assume that all the wireless links are subject to multipath propagation and that all the clusters of scatterers are close to the transmitters so that the scatters can be divided in $M$ clusters, each one being associated with a given user and its relay. Each cluster of scatterers has a mean angle of arrival and the cluster angle spread is assumed to be small compared to the spatial resolution of the antenna array. This assumption is valid in practice when the user and its relays are close to each other and no local scattering occurs around the base station antenna array. This assumption is typical in suburban environments [13], where the base transceiver station is on a tower or on the roof of a building. With these assumptions, the multipath propagation channels $h_{k, m}^{(S D)}$ can then be parameterized as [14]:

$$
h_{k, m}^{(S D)}=\sum_{l=1}^{L_{m}^{(S D)}} a_{k}\left(\theta_{m}\right) \beta_{l, m}^{(S D)} \approx a_{k}\left(\theta_{m}\right) \gamma_{m}^{(S D)},
$$

where $\theta_{m}$ is the mean angle of arrival of the $m$ th cluster, $a_{k}\left(\theta_{m}\right)=$ $\exp \left(-\jmath \pi(k-1) \sin \theta_{m}\right)$ is the response of the $k$ th antenna to the paths of the $m$ th cluster, $\beta_{l, m}^{(S D)}$ is the fading envelope of the $l$ th direct path between the $m$ th user and the base station, $L_{m}^{(S D)}$ being the corresponding number of multipaths, and $\gamma_{m}^{(S D)}=\sum_{l=1}^{L_{m}^{(S D)}} \beta_{l, m}^{(S D)}$. Similarly, the channel $h_{k, r, m}^{(R D)}$ can be expanded as:

$$
h_{k, r, m}^{(R D)}=\sum_{l=1}^{L_{r, m}^{(R D)}} a_{k}\left(\theta_{m}\right) \beta_{l, r, m}^{(R D)} \approx a_{k}\left(\theta_{m}\right) \gamma_{r, m}^{(R D)},
$$

where $\beta_{l, m}^{(R D)}$ is the fading envelope of the $l$ th path between the $r$ th relay of $m$ th user and the base station, $L_{r, m}^{(R D)}$ being the corresponding number of multipaths, and $\gamma_{r, m}^{(R D)}=\sum_{l=1}^{L_{r, m}^{(R D)}} \beta_{l, r, m}^{(R D)}$. Substituting (6) into (1), we get:

$$
x_{k, n}^{(S D)}=\sum_{m=1}^{M} a_{k}\left(\theta_{m}\right) \gamma_{m}^{(S D)} s_{n, m}+v_{k, n}^{(S D)} .
$$

Likewise, substituting (7) into (4), respectively, yields:

$$
x_{k, r, n}^{(R D)}=\sum_{m=1}^{M} a_{k}\left(\theta_{m}\right) \gamma_{r, m}^{(R D)} h_{r, m}^{(S R)} g_{r, m} s_{n, m}+v_{k, r, n}^{(S D)}
$$

\section{TENSOR MODELING}

Using the system model described in the last section, the received signal can be viewed as a three-way (tridimensional) array, with dimensions associated with space (receive antennas), slot (cooperative channel) and time (symbol period). In this section, we resort to the $\mathrm{CP}$ decomposition to model the received data in tensor form. As we will see later, the underlying tensor model has a powerful uniqueness property that allows the development of a blind receiver for joint channel estimation and symbol detection jointly exploiting spatial and cooperative diversities.

Let $\mathcal{X} \in \mathbb{C}^{K \times(R+1) \times N}$ be the third-order data tensor collecting the received signal at the base station organized in the following way:

$$
[\mathcal{X}]_{k, 1, n}=x_{k, n}^{(S D)}, \quad \text { and } \quad[\mathcal{X}]_{k, r, n}=x_{k, r-1, n}^{(R D)},
$$

where $k=1,2, \ldots, K, r=2,3, \ldots, R+1, n=1,2, \ldots, N$. In order to simplify the presentation of the tensor model, we omit the AWGN terms from the received signal equations throughout this section. Assuming that the channel is constant during $N$ symbol periods, a typical element of $\mathcal{X}$, denoted by $x_{k, r, n}=[\mathcal{X}]_{k, r, n}$, can be decomposed as:

$$
x_{k, n, r}=\sum_{m=1}^{M} a_{k}\left(\theta_{m}\right) h_{r, m} s_{n, m},
$$

where

$$
h_{r, m}= \begin{cases}\gamma_{m}^{(S D)} & \text { if } r=1 \\ \gamma_{r-1, m}^{(R D)} h_{r-1, m}^{(S R)} g_{r-1, m}, & \text { if } r>1\end{cases}
$$

The data tensor $\mathcal{X}$ can also be expressed as:

$$
\mathcal{X}=\sum_{m=1}^{M} \mathbf{A}_{\cdot, m} \circ \mathbf{H}_{\cdot, m} \circ \mathbf{S}_{\cdot, m},
$$


where $\circ$ denotes the outer product, $\mathbf{A} \in \mathbb{C}^{K \times M}$ contains the array response vectors, with $[\mathbf{A}]_{k, m}=a_{k}\left(\theta_{m}\right), \mathbf{H} \in \mathbb{C}^{(R+1) \times M}$ contains the channel vectors, with $[\mathbf{H}]_{r, m}=h_{r, m}$, and $\mathbf{S} \in \mathbb{C}^{N \times M}$ contains the symbol vectors, with $[\mathbf{S}]_{n, m}=s_{n, m}$. Equation (11) represent the CP decomposition $[9,10]$ of the received data tensor $\mathcal{X}$, with $\mathbf{A}$, $\mathbf{H}$ and $\mathbf{S}$ being the matrix factors of the decomposition. As shown in (13), the CP decomposition expresses a rank- $M$ data tensor as a sum of rank-1 component tensors, each one being given by the outer product of three vectors. The key property of model (11) is its essential uniqueness. In contrast to matrix (bilinear) decompositions the $\mathrm{CP}$ decomposition of higher-order tensors is unique of to trivial (scaling and permutation) indeterminacies under some conditions [15].

\subsection{Matrix representations}

We can also rewrite (11) in a matrix form. Define $\mathbf{X}_{k}$.. $\in$ $\mathbb{C}^{(R+1) \times N}$ the $k$ th first-mode slice, $\mathbf{X} . r . \in \mathbb{C}^{N \times K}$ the $r$ th second-mode slice and $\mathbf{X} \ldots n \in \mathbb{C}^{K \times(R+1)}$ the $m_{j}$-th third-mode slice. We have:

$$
\begin{aligned}
& \mathbf{X}_{k . .}=\mathbf{H} \operatorname{diag}_{k}[\mathbf{A}] \mathbf{S}^{T}, \\
& \mathbf{X}_{. r .}=\mathbf{S} \operatorname{diag}_{n}[\mathbf{H}] \mathbf{A}^{T}, \\
& \mathbf{X} . n=\mathbf{A} \operatorname{diag}_{r}[\mathbf{S}] \mathbf{H}^{T},
\end{aligned}
$$

where $\operatorname{diag}_{i}[\cdot]$ denotes the diagonal matrix formed with the $i$ th row of its matrix argument. The received data tensor $\mathcal{X} \in$ $\mathbb{C}^{K \times(R+1) \times N}$ can be "unfolded" into the form of three matrices $\mathbf{X}_{[1]} \in \mathbb{C}^{N(R+1) \times K}, \mathbf{X}_{[2]} \in \mathbb{C}^{K N \times(R+1)}$ and $\mathbf{X}_{[3]} \in \mathbb{C}^{(R+1) K \times N}$ by stacking columnwise the matrix slices as follows:

$$
\begin{gathered}
\mathbf{X}_{[1]}=\left[\begin{array}{c}
\mathbf{X}_{.1 .} \\
\vdots \\
\mathbf{X}_{.(R+1) .}
\end{array}\right], \quad \mathbf{X}_{[2]}=\left[\begin{array}{c}
\mathbf{X}_{\ldots 1} \\
\vdots \\
\mathbf{X}_{. N}
\end{array}\right], \\
\mathbf{X}_{[3]}=\left[\begin{array}{c}
\mathbf{X}_{1 \ldots} \\
\vdots \\
\mathbf{X}_{K} . .
\end{array}\right] .
\end{gathered}
$$

These matrices admit, respectively, the following factorizations:

$$
\begin{aligned}
& \mathbf{X}_{[1]}=(\mathbf{H} \diamond \mathbf{S}) \mathbf{A}^{T}, \\
& \mathbf{X}_{[2]}=(\mathbf{S} \diamond \mathbf{A}) \mathbf{H}^{T}, \\
& \mathbf{X}_{[3]}=(\mathbf{A} \diamond \mathbf{H}) \mathbf{S}^{T},
\end{aligned}
$$

where $\diamond$ denotes the Khatri-Rao (column-wise Kronecker) product.

\subsection{Uniqueness and its implications}

For $M \geq 2$, the essential uniqueness of the CP decomposition of $\mathcal{X}$ is assured when the Kruskal's condition is satisfied $[6,15]$ :

$$
k_{\mathbf{A}}+k_{\mathbf{H}}+k_{\mathbf{S}} \leq 2 M+2,
$$

where $k_{\mathbf{A}}$ denotes the k-rank of matrix $\mathbf{A}$, which corresponds to the greatest integer $k_{\mathbf{A}}$ such that every set of $k_{\mathbf{A}}$ columns of $\mathbf{A}$ is linearly independent. The essential uniqueness of the $\mathrm{CP}$ decomposition means that any alternative matrix set $\left\{\mathbf{A}^{\prime}, \mathbf{H}^{\prime}, \mathbf{S}^{\prime}\right\}$ satisfying (11) is related with the true matrix set by $\mathbf{A}^{\prime}=\mathbf{A} \Pi \boldsymbol{\Lambda}_{1}$, $\mathbf{H}^{\prime}=\mathbf{H} \boldsymbol{\Pi} \boldsymbol{\Lambda}_{2}$ and $\mathbf{S}^{\prime}=\mathbf{S} \boldsymbol{\Pi} \boldsymbol{\Lambda}_{3}$, where $\boldsymbol{\Pi} \in \mathbb{C}^{M \times M}$ is a column-permutation matrix and $\Lambda_{1}, \Lambda_{2}$ and $\Lambda_{3}$ are diagonal matrices such that $\Lambda_{1} \Lambda_{2} \Lambda_{3}=\mathbf{I}_{M}$.
Assuming that $\mathbf{A}, \mathbf{H}$ and $\mathbf{S}$ are full k-rank, condition (22) becomes:

$$
\min (K, M)+\min (R+1, M)+\min (N, M) \leq 2 M+2 .
$$

If the elements of the channel matrix $\mathbf{H}$ are statistically independent and drawn from an absolutely continuous distribution, then $\mathbf{H}$ is full k-rank with probability one, that is, $k_{\mathbf{H}}=\min (R+$ $1, M)$ [7]. In our model, such an assumption is valid when the user signals undergo independent fading channels. Moreover, in our model $\mathbf{A}$ is a Vandermonde matrix, which is full k-rank if it has distinct generators [8]. This assumption is true if the user signals arrive at the base station array with distinct directions of arrival. Note also that the symbol matrix $\mathbf{S}$ is full k-rank with high probability provided that $N$ is sufficiently large with respect to the modulation cardinality.

Under these assumptions, the uniqueness condition (23) can be used to easily determine, for fixed system design parameters, an upper bound on the number of active users to be jointly handled at the receiver. Similarly, condition (23) also help to choose "minimum" values for the system parameters (i.e. number $M$ of receive antennas, number $R$ of relays and data block length $N$ ) that cope with a target number of user channels (signals) to be estimated (detected). The flexibility on the choice of $K, N$ and $R$ provided by Kruskal's condition (23) is one of the main advantages of the proposed tensor modeling approach, since it leads to different tradeoffs involving the system parameters. For instance, it is reasonable to assume $N \geq M$. Under this assumption, the following can be deduced from (23):

- If $K \geq M$, then a single cooperative relay is enough to handle $M$ users;

- If $R \geq M-1$, then two base station receive antennas are enough to handle $M$ users.

On the other hand, if we assume that $K \geq M$ and $R \geq M-1$, then channel estimation and symbol detection can be accomplished with a very short data block of at least $N=2$ symbol periods. We can then conclude that the proposed tensor modeling approach covers a range of practical scenarios including: (a) the case where there is only one relay per user; (b) the case where we have less receive antennas than users, and (c) the case where very short data block are available for channel estimation.

\section{RECEIVER ALGORITHM}

We present a receiver algorithm for jointly and blindly estimate the channel parameters and the transmitted symbols in the considered cooperative diversity scheme. Specifically, we assume that channel state information is not available at the relays neither at the base station. The proposed receiver consists in fitting a $\mathrm{CP}$ tensor model to the received signal using the Levenberg-Marquardt (LM) algorithm [16].

The cost function to be minimized is given by:

$$
\begin{aligned}
J_{(\mathbf{A}, \mathbf{H}, \mathbf{S})} & =\sum_{k=1}^{K} \sum_{r=1}^{R+1} \sum_{n=1}^{N}\left|x_{k, r, n}-\sum_{m=1}^{M} a_{k}\left(\theta_{m}\right) h_{r, m} s_{n, m}\right|^{2} \\
& =\sum_{k=1}^{K} \sum_{r=1}^{R+1} \sum_{n=1}^{N}\left|r_{k, r, n}(\mathbf{A}, \mathbf{H}, \mathbf{S})\right|^{2}
\end{aligned}
$$

where $r_{k, r, n}(\mathbf{A}, \mathbf{H}, \mathbf{S})$ are the residuals. Define the global parameter vector concatenating all the unknowns as:

$$
\mathbf{p}=\left[\operatorname{vec}\left(\mathbf{A}^{T}\right), \operatorname{vec}\left(\mathbf{H}^{T}\right), \operatorname{vec}\left(\mathbf{S}^{T}\right)\right]^{T} \in \mathbb{C}^{F \times 1},
$$


where $F=M(K+R+N+1)$. We can rewrite (24) as:

$$
J_{\mathbf{p}}=\sum_{q=1}^{Q}\left|r_{q}(\mathbf{p})\right|^{2}=\mathbf{r}^{H}(\mathbf{p}) \mathbf{r}(\mathbf{p}),
$$

where $q=K(R+1)(n-1)+K(r-1)+k, Q=K(R+1) N$, and $\mathbf{r}(\mathbf{p})=\left[r_{1}(\mathbf{p}), \ldots, r_{Q}(\mathbf{p})\right]^{T} \in \mathbb{C}^{Q \times 1}$ is the vector or residuals. An approximation of the parameter vector $\mathbf{p}$ at the $(i+1)$-th iteration can be calculated from its approximation at the $i$-th iteration by $\mathbf{p}(i+$ 1) $=\mathbf{p}(i)+\Delta \mathbf{p}$, where the correction term $\Delta \mathbf{p}$ is a first-order linear approximation of the vector of residuals in the neighborhood of $\mathbf{p}(i)$ by a Taylor expansion. By minimizing $J_{(\Delta \mathbf{p})}, \Delta \mathbf{p}$ is found by:

$$
\Delta \mathbf{p}=-\left(\mathbf{J}^{H} \mathbf{J}+\lambda \mathbf{I}_{F}\right)^{-1} \mathbf{g},
$$

where $\mathbf{J} \doteq \mathbf{J}(\mathbf{p}(i)) \in \mathbb{C}^{Q \times F}$ is the Jacobian of $\mathbf{p}(i), \mathbf{g} \in \mathbb{C}^{F \times 1}$ is the gradient of $J_{\Delta \mathbf{p}}$, and $\lambda$ is a regularization term (damping factor) ${ }^{1}$. The update of the parameter vector is given by:

$$
\mathbf{p}(i+1)=\mathbf{p}(i)+\Delta \mathbf{p}
$$

Due to the partitioned structure of the parameter vector $\mathbf{p}$ defined in (25), we can write the Jacobian and gradient as a concatenation of three blocks $\mathbf{J}=\left[\mathbf{J}_{\mathbf{A}}, \mathbf{J}_{\mathbf{H}}, \mathbf{J}_{\mathbf{S}}\right]$ and $\mathbf{g}=\left[\mathbf{g}_{\mathbf{A}}^{T}, \mathbf{g}_{\mathbf{H}}^{T}, \mathbf{g}_{\mathbf{S}}^{T}\right]^{T}$, where the individual blocks are found using the compact expressions given in [16]. These expressions are not provided here due to lack of space.

\section{SIMULATION RESULTS}

In this section, some computer simulation results are provided for evaluating the performance of the proposed receiver under the following scenario. The wireless links are characterized by a frequency-flat Rayleigh fading with a path loss exponent equal to 3. The ratio of the distance between a user and its relays and the distance between the source and the destination being equal to 0.1 . The base station antenna array is composed of $K$ half-wavelength spaced antenna elements and BPSK modulation is assumed. The results represent an average over at least 100 independent channel and noise realizations. The noise variance is assumed to be the same at the base station and at all the relays.

Figure 1 shows the bit-error-rate (BER) versus the signal-to-noise-ratio (SNR) on the second hop (base station) provided by the proposed LM-based receiver with $M=2$ users, $N=16$ symbols, $K=2$ and 3 receive antennas, and $R=1$ and 2 relays per user. For comparison, it is also shown the BER provided by the ALS algorithm, as well as by the zero forcing $(\mathrm{ZF})$ receiver:

$$
\hat{\mathbf{S}}=\left[(\mathbf{H} \diamond \mathbf{A})^{\dagger} \mathbf{X}_{[3]}\right]^{T},
$$

assuming an instantaneous channel state information (CSI), that is, the matrices $\mathbf{H}$ and $\mathbf{A}$ are known perfectly. This figure shows that an increase on the number of receive antenna or relays decreases the BER. However, an increase of the number of relays provides higher gains than than an increase of the number of receive antenna, which means that the proposed receiver exploits both spatial and cooperative diversities, with the cooperative diversity providing further gains. Moreover, the LM algorithm provides BERs significantly smaller than the ones of the ALS algorithm and significantly higher than the ones of ZF-CSI method.

Fig. 2 shows the influence of the number $K$ of receive antennas on the BER. The results were obtained with the proposed LM-based

\footnotetext{
${ }^{1}$ In this work, the updating strategy of $\lambda$ follows that of [16]
}

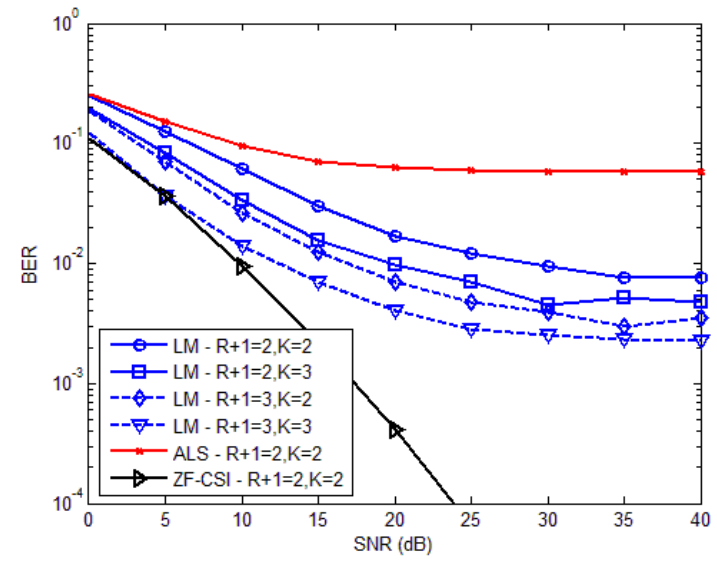

Fig. 1. BER versus SNR provided by the proposed LM-based receiver, the ALS algorithm and the ZF-CSI method.

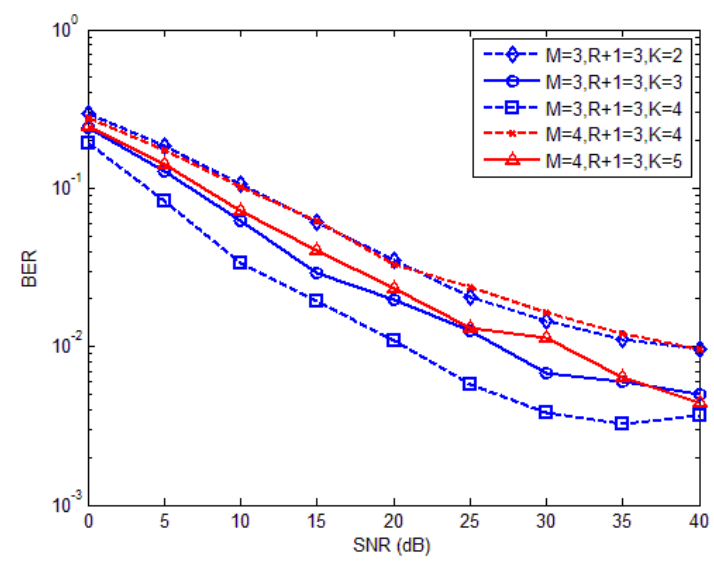

Fig. 2. BER versus SNR provided by the proposed LM-based method, for several values of the number $K$ of receive antennas.

receiver with $M=3$ and 4 users, $N=16, R=2$, and several values of $K$. It can be viewed that the BER is significantly decreased when $K$ is augmented. For instance, for $M=3$ and $B E R=10^{-2}$, making $K=4$ provides a SNR gain of approximately $18 \mathrm{~dB}$ with respect to the case $K=2$. Moreover, it should be highlighted that, for high SNRs, the proposed receiver provides BERs less than $10^{-2}$ for all the cases shown in Fig. 2.

The estimation of the array response matrix $\mathbf{A}$ is evaluated by means of the mean square error (MSE) of the estimated channel parameters, defined as:

$$
M S E=\frac{1}{N_{R} K M} \sum_{l=1}^{N_{R}}\left\|\mathbf{A}-\hat{\mathbf{A}}_{l}\right\|_{F}^{2},
$$

where $N_{R}$ is the number of Monte Carlo simulations, $\hat{\mathbf{A}}_{l}$ represents the matrix A estimated at the $l^{\text {th }}$ Monte Carlo simulation and $\|\cdot\|_{F}^{2}$ denotes the Frobenius norm. Fig. 3 shows the NMSE versus SNR provided by the proposed LM-based receiver and by the ALS algorithm with $M=3$ users, $N=16$ symbols, $K=3$ receive antennas and $R=2$ relays per user. For comparison, it is also 


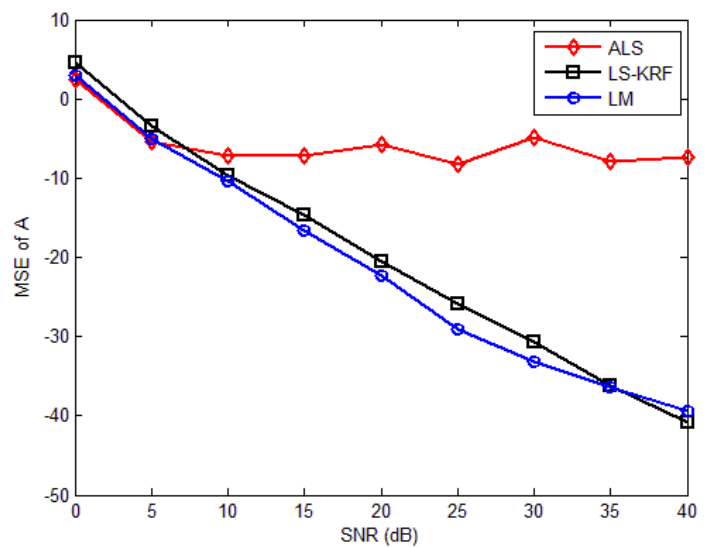

Fig. 3. MSE of A versus SNR provided by the proposed LM-based method, the ALS algorithm and the LS-KRF method.

shown the MSE provided by a supervised technique, denoted by least squares - Khatri-Rao factorization (LS-KRF) [11, 17], that computes a LS estimation of the matrix $(\mathbf{H} \diamond \mathbf{A})$ and, then, it uses this estimate to compute $\hat{\mathbf{H}}$ and $\hat{\mathbf{A}}$ by means of several singular value decompositions (SVD). It should be mentioned that, in Fig.3, the LS-KRF technique assumes that $M$ pilot symbols per user are used. From this figure, it can be concluded that the MSE provided by the proposed receiver is significantly smaller than the one obtained with the ALS algorithm and a bit smaller than the one obtained with the LS-KRF technique. It should also be remarked that proposed receiver is able to provide good channel estimates using the channel outputs measured during only 16 symbol periods and using only one known pilot symbol per user to remove the scaling ambiguity. The results concerning the estimation of the channel matrix are omitted due to lack of space.

\section{CONCLUSION}

In this work we have proposed a receiver that jointly and blindly estimate the channel gains, antenna array responses and transmitted symbols in a multiuser cooperative communication system. The proposed receiver exploits both the spatial and cooperative diversities to recover the transmitted symbols and consists in fitting a tensor model to the received signal using the LM algorithm. The underlying tensor model has a powerful uniqueness property that allows a great the flexibility on the choice of some system parameters, covering a range of practical scenarios including: the case where there is only one relay per user, the case where we have less receive antennas than users and the case where very short data blocks are available for channel estimation. Perspectives of this work include the extension of the proposed approach to multiple-input multiple-output (MIMO) cooperative systems with space-time coding.

\section{REFERENCES}

[1] P. A. Anghel and M. Kaveh, "Exact symbol error probability of a cooperative network in a Rayleigh-fading environment," IEEE Trans. Wireless Commun., vol. 3, no. 5, pp. 1416-1421, Sep. 2004.
[2] A. Sendonaris, E. Erkip, and B. Aazhang, "User cooperation diversity - part I: system description," IEEE Trans. Commun., vol. 51, no. 11, pp. 1927-1938, Nov. 2003.

[3] A. Sendonaris, E. Erkip, and B. Aazhang, "User cooperation diversity - part II: implementation aspects and performance analysis," IEEE Trans. Commun., vol. 51, no. 11, pp. 1939-1948, Nov. 2003.

[4] T. A. Tsiftis, G. K. Karaginnidis, S. A. Kotsopoulus, and F.-N. Pavlidou, "BER analysis of collaborative dual-hop wireless transmission," Electron. Lett., vol. 40, no. 11, pp. 679-680, May 2004.

[5] J. N. Laneman, D. N. C. Tse, and G. W. Wornell, "Cooperative diversity in wireless networks: efficient protocols and outage behavior," IEEE Trans. Inf. Theory, vol. 50, no. 12, pp. 3062-3080, Dec. 2004.

[6] N. D. Sidiropoulos, G. B. Giannakis, and R. Bro, "Blind PARAFAC receivers for DS-CDMA systems," IEEE Trans. Signal Process., vol. 48, no. 3, pp. 810-823, Mar. 2000.

[7] N. D. Sidiropoulos, R. Bro, and G. B. Giannakis, "Parallel factor analysis in sensor array processing," IEEE Trans. Signal Process., vol. 48, no. 8, pp. 2377-2388, Aug. 2000.

[8] N. D. Sidiropoulos and X. Liu, "Identifiability results for blind beamforming in incoherent multipath with small delay spread," IEEE Trans. Signal Process., vol. 49, no. 1, pp. 228-236, Jan. 2001.

[9] R. A. Harshman, Foundations of the PARAFAC procedure: Models and conditions for an "explanatory" multimodal factor analysis, UCLA Working Papers in Phonetics, 16th edition, Dec. 1970.

[10] J. D. Carroll and J.-J. Chang, "Analysis of individual differences in multidimensional scaling via an $\mathrm{N}$-way generalization of "Eckart-Young" decomposition," Psychometrika, vol. 35, no. 3, pp. 283-319, Sep. 1970.

[11] F. Roemer and M. Haardt, "Tensor-based channel estimation and iterative refinements for two-way relaying with multiple antennas and spatial reuse," IEEE Trans. Signal Process., vol. 58, no. 11, pp. 5720-5735, Nov. 2010.

[12] A. Y. Kibangou and A. L F. de Almeida, "Distributed PARAFAC based DS-CDMA blind receiver for wireless sensor networks," Proc. SPAWC 2010, Marrakech, Morocco, Jun. 2010.

[13] R. B. Ertel, P. Cardieri, K. W. Sowerby, T. S. Rappaport, and J. H. Reed, "Overview of spatial channel models for antenna array communication systems.," IEEE Personal Commun., vol. 5, no. 1, pp. 10-22, Feb. 1998.

[14] A.-J. van der Veen, "Algebraic methods for deterministic blind beamforming.," Proc. of IEEE, vol. 86, no. 10, pp. 1987-2008, Oct. 1998.

[15] J. Kruskal, “Three way arrays: Rank and uniqueness of trilinear decomposition with applications to arithmetic complexity and statistics," Linear Algebra and its Applications, vol. 18, pp. 95-138, 1977.

[16] G. Tomasi and R. Bro, "A comparison of algorithms for fitting the parafac model," Comp. Stat. Data Anal., vol. 50, pp. 1700-1734, 2006.

[17] C. A. R. Fernandes, G. Favier, and J. C. M. Mota, "Blind estimation of nonlinear instantaneous channels in multiuser CDMA systems with PSK inputs," in Proc. SPAWC 2008, Recife, Brazil, Jul. 2008. 